\title{
Sharing Model of Online Overseas Chinese Education Resource Based on Cloud Computing
}

\author{
http://dx.doi.org/10.3991/ijet.v8i6.3238 \\ Zhehuang Huang \\ Huaqiao University, China \\ Fujian Key Laboratory of the Brain-like Intelligent Systems, Xiamen, China
}

\begin{abstract}
In recent years, with the sustainable and fast development of chinese economy, there has been increasing interest in overseas chinese language. As an important way of overseas chinese education, online education has been drawing more and more attention due to its simplicity and convenience. But at present, the existing chinese education resource service model is simply assigned the resources to users which can not effectively meet the practical needs of users. How to provide a personalized service is the key problem to be solved. In this paper, we proposed a sharing model of chinese education resource based on cloud computing. There are three mainly works in this paper. Firstly, user vector space is constructed based on user personal information. Secondly, synergetic neural network is presented to user group recognition. Finally, sharing model based cloud computing is presented and implemented. The proposed model in this paper provide a good practicability and a promising future for overseas chinese education.
\end{abstract}

Index Terms-overseas chinese education, cloud computing, synergetic neural network, user modeling.

\section{INTRODUCTION}

With the great development of Chinese economy, the influence of Chinese education becomes more and more larger. At present, many countries and regions have established Confucius institute and Confucius classroom. Overseas chinese education $[1,2]$ has made tremendous contributions. However, due to historical reasons and international environment, the overseas Chinese teaching resources are relatively scattered and weak, which will great influence the development of overseas Chinese education. How to better share the Chinese overseas resources and provide personalized service is the key problem urgently to be solved at present.

In recent years, with the development and popularity of network, information services everywhere. At the same time, there have been some problems such as rising demand, massive data growth, and heterogeneous distribution of resources. Cloud computing[3,4] as a new shared infrastructure approach can enable large amounts of data and processor resources to work together, so as to provide users with a variety of storage and computing resources. Cloud computing can combine the database into a onestop search interface together.

At present, the existing Chinese education resource is statically assigned to users which can not meet the needs of users. User modeling method[5-7] should be automatically constructed according to the user's browsing content and browsing behavior. A key problem of intelligence user server model is how to classifier and indentify the user groups. It can offer better service only when the user groups can be effectively identified.

Synergetics [8-10] is the science proposed by Haken to explain the phase transition and self-organization in nonequilibrium system. In the late 1980s, Haken proposed to put synergetic theory into a new field: synergetic information processing, which includes synergetic neural network, synergetic information theory and synergetic computer, etc[11-13] .

In this paper, we presents a sharing model of Chinese education resource based on cloud computing. This paper is organized as follows. Firstly, the oval sharing model is presented. Secondly, some key algorithm of our proposed model is implemented. Finally some discuss and conclusions are given on the model.

\section{THE RESOURCE SHARING BASED ON CLOUD COMPUTING}

In this section, we present a personalized service model based on cloud computing. Firstly, we can build user information vector based on the characteristics of users. Secondly, we proposed a user group classification algorithm based on synergetic neural network .Finally, A personalized information sharing model based on the cloud computing is presented. The sharing model is shown in figure 2 .

\section{A. User information vector}

The user information can be viewed as a vector, so we can better describe the relationship between user groups. The user information space is show as follow.

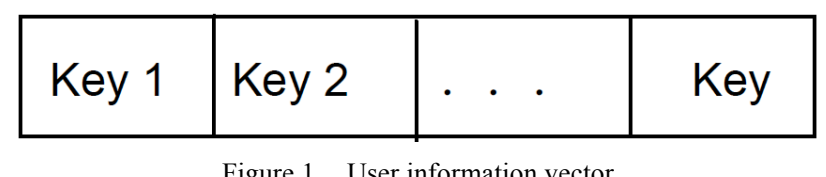

\section{B. User group classification}

In synergetics, high dimension and nonlinear problem can be described as a set of low-dimension nonlinear equations, which focuses on the research of the qualitative change of macro-feature in complex system. The basic principle of synergetic neural network is that the pattern recognition procedure can be viewed as the competition progress of many order parameters. The strongest order parameter will win by competition and desired pattern will be recognized. User identification is a classification problem, we use synergetic neural network to realize the user classification. 


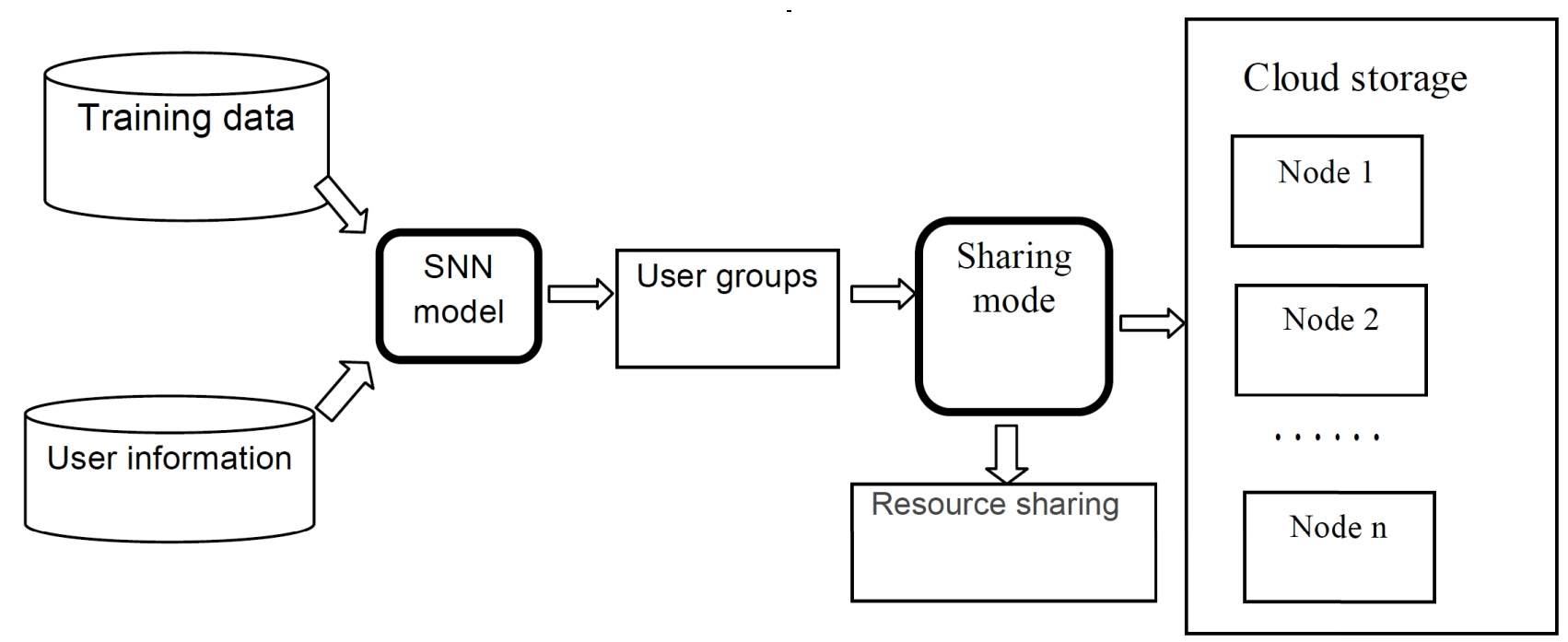

Figure 2. . Sharing mode based on cloud computing

\section{Cloud service model}

The cloud service model consist cloud monitor, user groups and server pool. Cloud monitor is responsible for managing user groups and server pool. Nodes in server pool are responsible for providing servers. When computers online apply for joining the system, monitor adds it to the server pool. If one node is in error, monitor deletes it from the server pool. Monitor adds user group to user group's pool. And all the results are summarized and exported to users.

\section{THE IMPLEMENTED OF OUR PROPOSED MODEL}

\section{A. The comstruction of user vector space and similarity measure}

TF-IDF (term frequency-inverse document frequency) $[14,15]$ is a commonly used weighting technique for information retrieval and text mining. TF-IDF $[5,6]$ is a statistical method used to assess the degree of importance of a word for one of the documents in a set of files or a corpus. The process of constructing the TF-IDF vector is described below.

Supposed the weight vector for document $d$ is $v_{d}=\left[w_{1 d}, w_{2 d} \mathrm{~L} w_{N d}\right]$, then $w_{t d}=\mathrm{TF}_{t d} \cdot \mathrm{IDF}_{t d}$

\section{B. Synergetic neural network (SNN) model}

An unrecognized pattern, $q$, is constructed by a dynamic process which translates $q$ into one of prototype pattern vectors $v_{k}$ through status $q(t)$, namely, this prototype pattern is closest to $q(0)$.The process is described as following equation: $q \rightarrow q(t) \rightarrow v_{k}$

Dynamic equation can be given for a unrecognized patter is:

$$
\dot{\xi}_{k}=\lambda_{k} \xi_{k}-B \sum_{k^{\prime} \neq k} \xi_{k^{\prime}}^{2} \xi_{k}-C\left|\sum_{k^{\prime}=1}^{M} \xi_{k^{\prime}}^{2}\right| \xi_{k}
$$

Where $\xi_{k}$ satisfies initial condition: $\xi_{k}=v_{k}^{+} q(0)$. A method for label semantic roles using Synergetic neural network technique is given as Figure 3 .

Order parameter reflects the similarity between user groups, the order parameters of ${ }^{d}$ and query $q$ can be calculated as:

$$
\xi_{j}=\frac{\sum_{i=1}^{N} w_{i, j} w_{i, q}}{\sqrt{\sum_{i=1}^{N} w_{i, j}^{2}} \sqrt{\sum_{i=1}^{N} w_{i, q}^{2}}}
$$

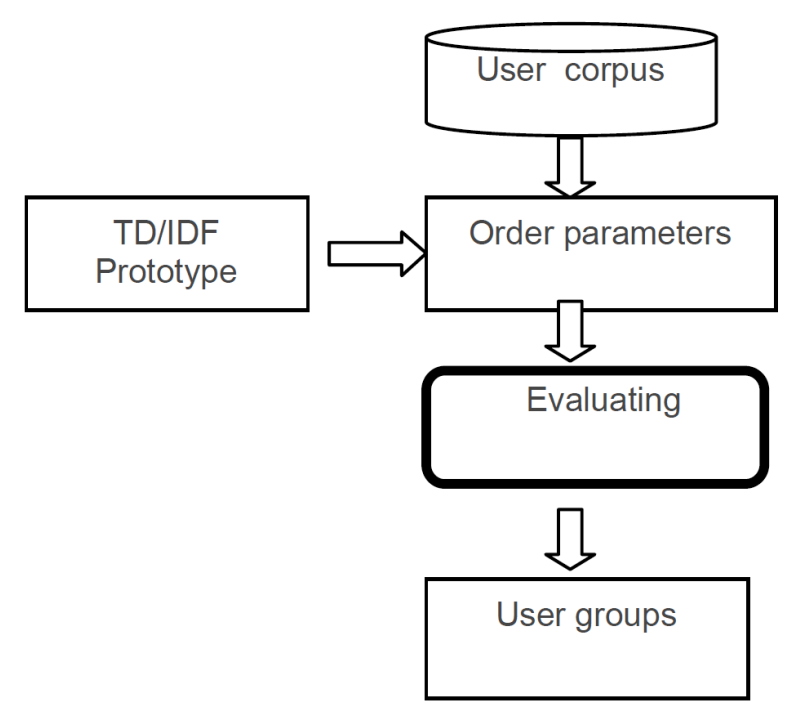

Figure 3. The users model based on synergetic neural network 


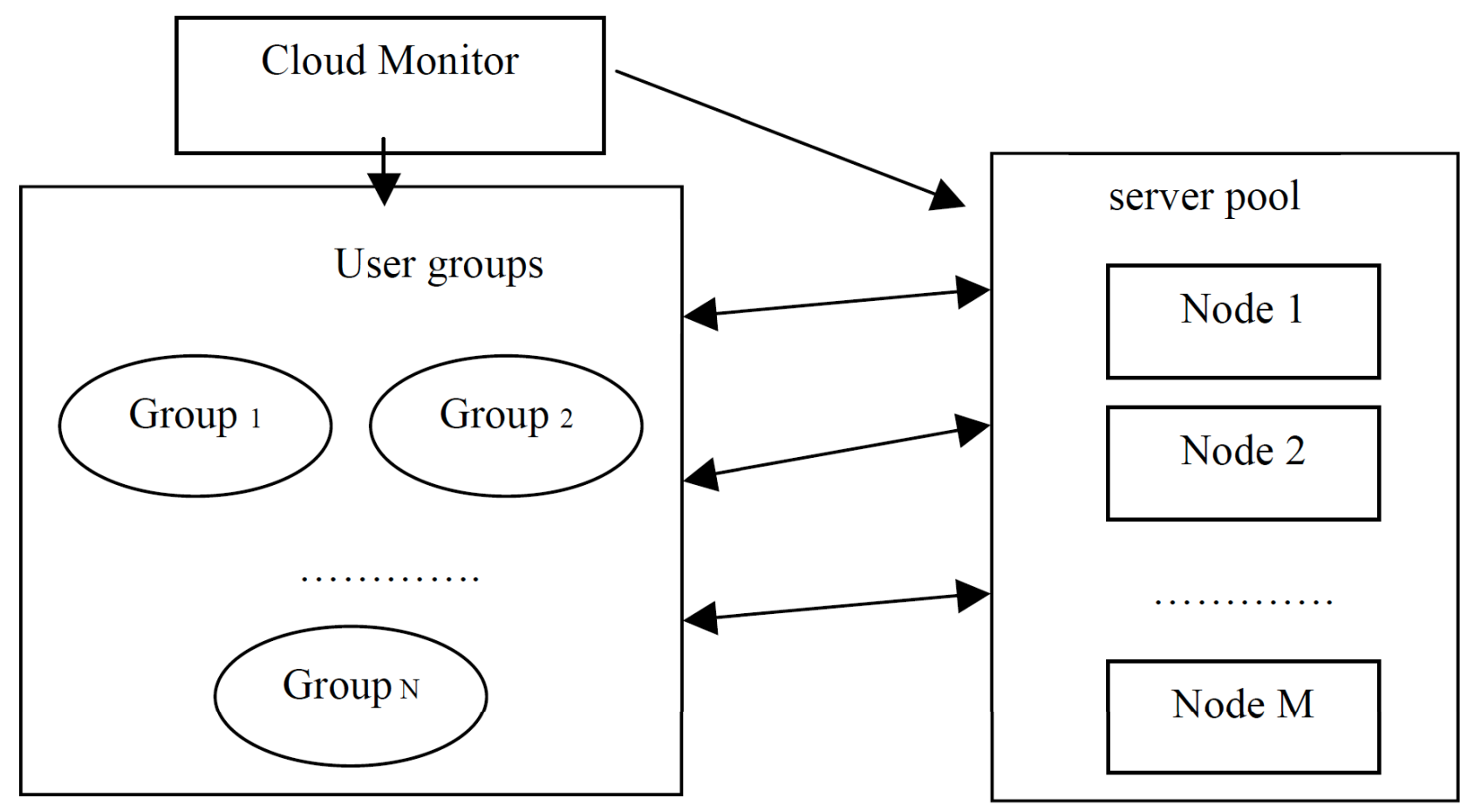

Figure 4. The realization of cloud computing model

\section{The realization of cloud computing storage}

Cloud node monitoring process is responsible for managing cloud nodes. Any computer on the network can join the cloud storage. The computer is joined to server pool by cloud node monitoring process. The cloud service model is shown as Fig.4.

The overall implementation process can be expressed as Algorithm 1.

1) TF-IDF algorithm is used to construct user vector space;

2) Calculate the order parameter based on according to the above formula (2);

3) Set the attention parameters $B, C$ and non- attention parameter $\lambda_{k}$;

4)We can obtain the best user group through the evaluating of order parameter equation according to the above formula (1).

5) Cloud service model is constructed and implemented.

6) Resource sharing based on personal information.

Algorithm 1. Overseas Chinese education resource Sharing based on cloud computing

\section{CONClusions}

In this paper, we present a sharing model of Chinese education resource based on cloud computing. This is the first effort to introduce cloud computing and SNN model to Chinese education resource Sharing. The proposed model provides a good practicability and a promising future for Chinese education resource sharing. We got the following conclusions.
1) The model based on cloud computing can effectively achieve the purpose of resource sharing Chinese education.

2) Synergistic neural network used to user group classification can quickly determine user groups.

\section{REFERENCES}

[1] D Starr. "Chinese Language Education in Europe: the Confucius Institutes". European Journal of Education,vol 44, no 1, Part I. 2009, pp 65-82.

[2] Yang, R. "Soft Power and Higher Education: An Examination of China's Confucius Institutes. Globalisation", Societies and Education, vol8 no 2, 2010, pp 235-245.

[3] Michael Armbrust, Armando Fox, Rean Griffith,Anthony D. Joseph, Randy Katz, Andy Konwinski,Gunho Lee, Dav id Patterson, "Ariel Rabkin, Ion Stoica,and Matei Zaharia". A View f Cloud Computing. communications of the ac $\mathrm{m}$. Vol.53,no. 4,2010, pp. 51-58.

[4] Mladen A. Vouk, "Cloud Computing-Issues, Research and Implementations, " Journal of Computing and Information Technology, 2008(4), pp.235-246.

[5] Navigli R.,Velard P.,Gangemi A. "Ontology learning and its application to automated terminology translation".IEEE Intelligent Systems, 18(1), 2003, pp. 22-31. http://dx.doi.org/10.1109/ MIS.2003.1179190

[6] Vijayan Sugumaran,Veda C.Storey. "Ontologies for conceptual modeling:their creation,use, and management”.Data \& Knowledge Engineering, 42(3), 2002, pp. 51-271. http://dx.doi.org/10.1016/ S0169-023X(02)00048-4

[7] Kerschberg,L.,Kim,W.,Scime,A. “A Semantic Taxonomy_Based Personalizable Meta-Search Agent”. Proceedings of 2th International Conference on Web Information System Engeering.Kyoto, Japan, 2001, pp.53-62.

[8] Haken. "Synergetic Computers and Cognition-A Top-Down Approach to Neural Nets". Springer-Verlag, Berlin , 1991.

[9] Weizhi Wang, Lei Pan and Binghan Liu. "Synergetic Method of Traffic State Recognition Based on Manifold Learning” ,In: Proceedings of the IEEE International Conference on Automation and Logistics, Shenyang, China, 2009, pp. 587-591. 


\section{SHORT PAPER}

\section{SHARING MOdel of ONLINE OverseAs CHINESE EdUCATION RESOURCE BASED ON ClOUd COMPUTING}

[10] Zhenhua Jiang , Roger A. Dougal. "synergetic control of power converters for pulse current charging of advanced batteries from a fuel cell power source". IEEE Transactions on Power Electronics, 19(4), 2004, pp. 1140-1150. $\quad$ http://dx.doi.org/10.1109/ TPEL.2004.830044

[11] WangGang,Gao Yang,Xia Jie. "Quick train of artificial neural network based on diffierential evolution". Chinese Journal of Management, 2(4), 2005,pp.450-454.

[12] Xiuli Ma, Shuang Wang, Licheng Jiao. " Robust Classification of Immunity Clonal Synergetic Network Inspired by Fuzzy Integral". In: Jun Wang, Xiaofeng Liao, Yi Zhang (Eds.), Lecture Notes in Computer Science, Springer, Berlin. 3497, 2005, pp.26-31.

[13] Gao Jun, Dong Huo-Ming, Shao Jing,et al. "Parameters optimization of synergetic recognition approach". Chinese Journal of Electronics, 14(2), 2005, pp.192-197.

[14] Huang Cheng Hui, Yin Jian, Hou Fang. "A Text Similarity Measurement Combining Word Semantic Information with TF/IDF Method". Chinese journal of computers,34(5),2011, pp.856-864.
[15] Ramiz M A. "A new sentence similarity measure and sentence based extractive technique for automatic text summarization". Expert Systems with Applications. 36(4),2009, pp.7764-7772. http://dx.doi.org/10.1016/j.eswa.2008.11.022

\section{AUTHOR}

Zhehuang Huang is a lecturer in the School of Mathematics Sciences, Huaqiao University. His research interest covers intelligent algorithm, machine learning and data fusion.

This work was supported by the National Natural Science Foundation of China (Grant No. 61005052), the Fundamental Research Funds for the Central Universities (Grant No. 2010121068) and the Natural Science Foundation of Fujian Province of China (Grant No.2011J01369), the science and technology project of Quanzhou (Grant No.2012Z91). Submitted 08 October 2013. Published as re-submitted by the author 08 November 2013. 